\title{
THE INFLUENCE OF CIRCADIAN RHYTHMS ON SUDDEN UNEXPECTED DEATH IN EPILEPSY
}

\author{
Vera C. Terra', Hélio R. Machado', Américo C. Sakamoto', Ricardo M. Arida², \\ Carla A. Scorza $a^{3}$ Marly de Albuquerque $e^{3}$ Esper A. Cavalheiro ${ }^{3}$, Fulvio A. Scorza ${ }^{3}$
}

Patients with epilepsy are at two- to three-fold higher risk of dying prematurely compared with healthy individuals, being the most frequent epilepsy-related cause of death the sudden unexpected death in epilepsy (SUDEP)'. SUDEP is defined as sudden, unexpected, witnessed or unwitnessed, nontraumatic and nondrowning death in patients with epilepsy. Documented status epilepticus (SE) is excluded in SUDEP, and post mortem examination does not reveal any cause of death ${ }^{2}$. Risk factors for SUDEP include epilepsy refractoriness, presence of generalized tonic-clonic seizures, polytherapy with antiepileptic drugs, young age, duration of the seizure disorder ranging from 15 to 20 years, and early epilepsy onset ${ }^{1,3}$. The pathophysiological causes of SUDEP are unknown, but it is very probable that cardiac abnormalities during and between seizures might play a potential role in the event ${ }^{1,3}$. In recent years, some studies have indicated that sudden cardiac death might follow circadian distribution ${ }^{4}$; however, to our knowledge, there is no data describing a possible relationship between daily, weekly or seasonal variation and SUDEP.

The present report shows a SUDEP case that could be associated with circadian distribution.

\section{CASE}

A 15 year-old boy with poorly controlled epilepsy, no perinatal neurological problems, and normal neurological and cognitive development presented with seizures since the age of three. Between three and ten years of age, seizures occurred sporadically, but they became clinically refractory to treatment thereafter. Patient described daily complex motor seizures, which occurred almost only during sleep. Seizures frequently evolved to tonic and tonic-clonic generalization. Clinical and neurological examinations were normal. Brain MRI scan gave evidence of volumetric reduction in the left temporal pole. Video-EEG re- vealed rare interictal frontal and rolandic spikes and complex partial seizures with bilateral frontal and temporal theta activity. Recent blood serum chemistry was normal for glucose, renal, hepatic and hematological functions. Levels of serum antiepileptic drugs were not available. Although he had been on polytherapy (oxcarbazepine, lamotrigine and topiramate), he experienced two to three seizures per night and took no other medications.

He was last seen alive at $7 \mathrm{AM}$ of a July Monday morning and continued sleeping. At 11 AM, he was found dead in bed, lying prone, with conjunctiva hyperemia. No post mortem examination was done. No signs of an infectious process or any other concomitant disease were noted, either. The patient was considered to have died of SUDEP.

\section{DISCUSSION}

This report describes circadian distribution (i.e. hours of the day, seasonal variation and days of the week) that could be involved in a SUDEP case. Recognition of these patterns could help develop the concept of cardiovascular events "triggering". As research in this field must focus on the potential prevention of SUDEP, a number of arguments might be put forward.

Firstly, colder temperatures might explain the SUDEP case described here because patient died during winter and it is possible that this factor contributed to a SUDEP event. Exposure to low temperatures is well-established as one of the risk factors in cardiac mortality ${ }^{4}$, and our group reported that winter temperatures may lead to some cardiovascular abnormalities and hence SUDEP ${ }^{5}$. This hypothesis has been experimentally evaluated and we demonstrated that low temperatures clearly increase the heart rate of rats with epilepsy, suggesting that cold weather could be considered an important risk factor in cardiovascular abnormalities and therefore sudden cardiac death in epi-

\footnotetext{
INFLUÊNCIA DO RITMO CIRCADIANO NA MORTE SÚBITA INESPERADA EM EPILEPSIA

'Departamento de Neurologia, Psiquiatria e Psicologia, Faculdade de Medicina de Ribeirão Preto, Universidade de São Paulo, Ribeirão Preto SP, Brazil; ${ }^{2}$ Departamento de Fisiologia, Universidade Federal de São Paulo/Escola Paulista de Medicina (UNIFESP/EPM), São Paulo SP, Brazil; ${ }^{3}$ Disciplina de Neurologia Experimental, Universidade Federal de São Paulo/Escola Paulista de Medicina (UNIFESP/EPM), São Paulo SP, Brasil. Financial Support: FAPESP, CInAPCe-FAPESP and CNPq.
}

Received 31 October 2008. Accepted 25 February 2009.

Dra. Vera C. Terra - Departamento de Neurologia / CIREP / Hospital das Clínicas de Ribeirão Preto - Avenida Bandeirantes SN - $14048-900$ Ribeirão Preto SP - Brasil.E-mail: vctbusta@rnp.fmrp.usp.br 
lepsy ${ }^{6}$. Following this reasoning, colder temperatures have been associated with an increase in vascular resistance, coronary vasospasm, blood pressure, and hemostasis ${ }^{4}$.

A second argument in favour of SUDEP in the case reported here is the fact that our patient died on a Monday. This finding is in agreement with available evidence that suggests a peak in the incidence of cardiovascular events on Mondays compared with others days ${ }^{7}$. Peckova and colleagues ${ }^{7}$ explored weekly variation in 6603 outof-hospital cardiac arrests during an 8-year period and observed a higher incidence of sudden death on Monday compared with the other days. Arntz and co-workers $^{4}$ analyzed the emergency medical system data of Berlin (West) from 1987-1991 with respect to all consecutive sudden deaths in subjects older than 18 years and found that a maximum of events occurred on Mondays ( $n=3721)$. This corresponded to a relative increase of $18.3 \%$, which was more pronounced in young patients and men compared with older patients and women, respectively.

The time of day when sudden cardiac deaths occur is another important issue. In the present case, the patient was last seen alive at $7 \mathrm{AM}$ and was found dead around 11 AM. This observation is in agreement with Muller and colleagues ${ }^{8}$, who analyzed the time of day of sudden cardiac death as indicated by death certificates of 2203 individuals dying out of the hospital. Their data revealed a prominent circadian variation of sudden cardiac death, with a low incidence during the night and an increased incidence from 7 to 11 A.M. Willich and colleagues ${ }^{9}$ analyzed the time of day when sudden cardiac deaths occurred and there was higher incidence of cardiac deaths between 7 and 9 AM, and the risk of such event was at least $70 \%$ higher during this peak period compared with the average risk during other periods of the day. More recently, a large study showed that the time of day with higher incidence of sudden deaths was between 6 and $10 \mathrm{AM}^{4}$.

Although the patient described here presented some risk factors that might have made him a potential candidate for SUDEP, our study shows that a possible role of circadian variation in the occurrence of this event should not be neglected. The present data could be sustained by Tomson's group, who demonstrated a circadian vari- ation in heart-rate variability in localization-related epilepsy ${ }^{10}$. To sum up, we would like to raise the possibility that circadian variation could underlie some processes that culminate in SUDEP and that heart failure could have a significant role in this mechanism. However, a clear relationship between epilepsy, cyclic patterns, and SUDEP still needs to be demonstrated in both experimental and human conditions.

\section{REFERENCES}

1. Stollberger C, Finsterer J. Cardiorespiratory findings in sudden unexplained / unexpected death in epilepsy (SUDEP). Epilepsy Res 2004;59:51-60.

2. Nashef L. Sudden unexpected death in epilepsy: terminology and definitions. Epilepsia 1997;38(Suppl):S6-S8.

3. Tomson T, Walczak T, Sillanpaa M, et al. Sudden unexpected death in epilepsy: a review of incidence and risk factors. Epilepsia 2005;46:54-61.

4. Arntz HR, Willich SN, Schreiber C, Brãggemann T, Stern R, Schultheiss HP. Diurnal, weekly and seasonal variation of sudden death. Population based analysis of 24,061 consecutive cases. Eur Heart J 2000;21:315-320.

5. Scorza FA, de Albuquerque M, Arida RM, Cavalheiro EA. Sudden unexpected death in epilepsy: are winter temperatures a new potential risk factor? Epilepsy Behav 2007;10:509-510.

6. Sonoda EYF, Colugnati DB, Scorza CA, et al. Is cold the new hot in sudden unexpected death in epilepsy? Effect of low temperature on heart rate of rats with epilepsy. Arq Neuropsiquiatr 2008;66:848-852.

7. Peckova M, Fahrenbruch CE, Cobb LA, Hallstrom AP. Weekly and seasonal variation in the incidence of cardiac arrests. Am Heart J 1999;137:512-515.

8. Muller JE, Ludmer PL, Willich SN, et al. Circadian variation in the frequency of sudden cardiac death. Circulation 1987;75: 131-138.

9. Willich SN, Levy D, Rocco MB, Tofler GH, Stone PH, Muller JE. Circadian variation in the incidence of sudden cardiac death in the Framingham Heart Study population. Am J Cardiol 1987; 60:801-806.

10. Persson H, Kumlien E, Ericson M, Tomson T. Circadian variation in heart-rate variability in localization-related epilepsy. Epilepsia 2007;48:917-922. 